\title{
Aspectos genéticos de curvas de probabilidade de postura em codornas
}

\author{
Robson Marcelo Rossi ${ }^{1}$, Elias Nunes Martins ${ }^{2}$
}

\begin{abstract}
${ }^{1}$ Departamento de Estatística, UEM.
2 Departamento de Zootecnia, UEM.

RESUMO - Neste trabalho foram avaliados os componentes de covariância e as herdabilidades dos parâmetros da curva de produção de ovos em codornas em um modelo de duplo estágio: o primeiro estágio composto pelo ajustamento de uma curva não-linear e o segundo, pela avaliação dos parâmetros genéticos obtidos por meio do modelo animal. Foram utilizados registros individuais diários de postura até 90 dias, a contar do primeiro ovo no lote, de 308, 374 e 378 aves de três linhagens. Dentro de cada linhagem, não houve diferenças entre os componentes de covariância nem herdabilidades para as rações contendo diferentes níveis de energia ( 2.900 ou $2.500 \mathrm{kcal} / \mathrm{kg}$ de EM), indicando que não houve heterogeneidade de variância. Entretanto, os valores de correlações genéticas foram na ordem de 0,53 a 0,65 para o parâmetro $\alpha$ e de 0,28 a 0,30 para $\beta$, indicando haver interação genótipo $\times$ ambiente. As estimativas de herdabilidade, respectivamente, nas três linhagens, para os parâmetros $\alpha$ e $\beta$ na dieta de alta energia foram 0,21 e 0,$50 ; 0,12$ e 0,49 ; e 0,12 e 0,48 , e na dieta de baixa energia, 0,23 e 0,$50 ; 0,13$ e 0,50 ; e 0,10 e 0,47 . As estimativas dos componentes de covariância e herdabilidades foram diferentes entre as linhagens, para os dois parâmetros da curva. Uma linhagem apresentou maior herdabilidade para o parâmetro $\alpha$, o que indica maior potencial para mudanças por seleção para a produção na fase inicial de postura. Considerando o parâmetro $\beta$, as três linhagens apresentam o mesmo potencial.
\end{abstract}

Palavras-chave: análise bayesiana, curvas não-lineares de produção, dados binários correlacionados, modelo animal, parâmetros genéticos

\section{Genetic aspects of laying probability curves in quails}

\begin{abstract}
It was evaluated in this work the components of (co)variance and heritability of the parameters of egg production curve in quails in a model of double stages: the first stage is composed of the adjustment of a nonlinear curve, and the second is composed of the assessing of the genetic parameters obtained by the animal model. It was used daily individual records from laying to 90 days, starting with the first egg in the batch, of 308, 374 and 378 birds from three lines. In each strain, there was no difference between the components of (co)variance neither heritability for rations containing different energy levels $(2,900$ or $2,500 \mathrm{kcal} / \mathrm{kg} \mathrm{ME})$, indicating that there was no heterogeneity of variance. However, values of genetic correlations were in the order from 0.53 to 0.65 for the $\alpha$ parameter and, for $\beta$, from 0.28 to 0.30 , indicating one existing genotype $\times$ environment interaction. Estimates of heritability, respectively in the three lines, for the $\alpha$ and $\beta$ parameters in the high energy diet were 0.21 and $0.50,0.12$ and $0.49,0.12$ and 0.48 , and for the low energy diet 0.23 and $0.50,0.13$ and $0.50,0.10$ and 0.47 . Estimates of the components of (co)variance and heritability were different among the lines, for the two curve parameters. One line showed higher heritability for the $\alpha$ parameter, indicating greater potential for changes by selection for production at the initial stage of laying. Considering the $\beta$ parameter, the three lines have the same potential.
\end{abstract}

Key Words: animal model, bayesian analysis, correlated binary data, genetic parameters, nonlinear production curves

\section{Introdução}

Em estudos de melhoramento genético, é comum o tipo de estrutura de dados longitudinais, além da estrutura de parentesco entre indivíduos e, nessas situações, Gianola (2001) relata que modelos hierárquicos ou em múltiplos estágios podem ser assumidos para tais situações.

A utilização de dados longitudinais em vez de dados acumulados pode produzir melhores resultados, pois consideram a variação ao longo da trajetória, permitindo obter valores de herdabilidade mais representativos (Sapp et al., 2005).

A busca por modelos que refletem de modo satisfatório a variabilidade nos dados levam a diferentes abordagens. Dentre as metodologias utilizadas para a estimação de parâmetros genéticos e fenotípicos, destacam-se: o procedimento de estimação pela Máxima Verossimilhança Restrita (Patterson \& Thompson, 1971), que, segundo Wang et al. (1994)

Recebido em 12/2/2009 e aprovado em 28/8/2009.

Correspondências devem ser enviadas para: rmrossi@uem.br 
e Resende (2002), apresenta deficiências; o procedimento por modelos de Regressão Aleatória (Schaeffer, 2004; Kranis et al., 2007), cuja principal característica é modelar a covariância no tempo e os Modelos Lineares Generalizados (Nelder \& Wedderburn, 1972). A abordagem Bayesiana (Gianola \& Fernando, 1986) pode ser utilizada de forma alternativa e, além de fornecer estimativas precisas e acuradas dos componentes de (co)variância, tende a conduzir também à maior aproximação entre ganhos genéticos preditos e realizados com a seleção, mesmo para modelos de grande complexidade; permite a simultânea estimação dos parâmetros genéticos, efeitos de ambiente e valores genéticos de maneira satisfatória, mesmo para amostras pequenas.

Apesar de o Brasil estar entre os três maiores produtores mundiais de codornas (Minvielle, 2004), são poucas as instituições que trabalham com essa espécie animal, e principalmente com avaliações genéticas. Segundo Martins (2002a), os programas de melhoramento genético devem ser realizados da forma mais eficiente, utilizando metodologias modernas e adequadas, para que a seleção resulte em vantagens. No caso da produção de ovos, a eficiência do processo de seleção é em parte devida à escolha adequada da função que descreve o comportamento da postura.

Nesse sentido, no presente trabalho os objetivos foram avaliar, em três linhagens distintas de codornas de postura, os parâmetros da curva de produção de ovos, além de obter e comparar estimativas dos componentes de covariância e parâmetros genéticos para cada linhagem e entre as mesmas.

\section{Material e Métodos}

O experimento foi realizado no setor de coturnicultura da Fazenda Experimental de Iguatemi, pertencente à Universidade Estadual de Maringá, Paraná, Brasil, no período de agosto de 2006 a janeiro de 2007, com a utilização de 308, 374 e 378 codornas de postura (Coturnix coturnix japonica) das linhagens amarela, azul e vermelha (cor das anilhas), respectivamente, em desenvolvimento no local desde 2002.

Foram efetuadas incubações com ovos coletados em dois períodos de oito dias, gerando dois grupos de eclosão distintos. Os ovos foram identificados por pai e mãe, de tal forma que foi possível a identificação da genealogia do pintainho ao nascimento. As aves foram anilhadas e criadas em piso com cama, recebendo ração para crescimento conforme recomendação do NRC (1994).
Aos 28 dias de idade, foi realizada a sexagem, e as fêmeas foram divididas em dois grupos, de tal forma que cada matriz teve filhas nos dois grupos. Nessa idade, as aves foram alojadas em gaiolas individuais e cada grupo passou a ser alimentado com um dos tipos de ração: com nível preconizado de energia (2.900 kcal/kg de energia metabolizável), em 163, 186 e 201 aves, respectivamente, das linhagens amarela, azul e vermelha, e de baixa energia $(2.500 \mathrm{kcal} / \mathrm{kg}$ de energia metabolizável), em 145, 188 e 177 aves, respectivamente, das linhagens amarela, azul e vermelha.

Os dados de postura foram coletados a partir da primeira postura observada no lote e seguiu-se até o 90 o dia de observação.

Foi definido que $Y_{i j}$ é o valor observado da postura (= 1 se a ave pôs ovo, ou $=0$ se não pôs ovo) no dia $j$, do animal $i$, em que $j=1,2, \ldots, \mathrm{J}(=90)$ e $i=1,2, \ldots, \mathrm{n}(=308$, 374 e 378 , respectivamente, para as linhagens amarela, azul e vermelha).

Considerando a produção diária de ovos, um modelo de efeitos aleatórios normalmente distribuídos com função de ligação logística foi utilizado no primeiro estágio de análise, como descrito a seguir.

Primeiro estágio: considerando a probabilidade de postura $\left(\theta_{i j}\right)$, a função de probabilidade de $Y_{i j}$ foi dada por:

$$
\mathrm{f}\left(Y_{i j}=y_{i j}\right)=\theta_{i j}^{y_{i j}}\left(1-\theta_{i j}\right)^{1-y_{i j}}= \begin{cases}\theta_{i j} ; & \text { para } y_{i j}=1 \\ 1-\theta_{i j} ; & \text { para } y_{i j}=0\end{cases}
$$

A função de verossimilhança para os $j$-ésimos dias foi dada por:

$$
\begin{aligned}
& L(\theta)=\prod_{i=1}^{n} \prod_{j=1}^{J} \theta_{i j}^{y_{i j}}\left(1-\theta_{i j}\right)^{1-y_{i j}} \text {, e seu logaritmo por: } \\
& l(\theta)=\sum_{i=1}^{n} \sum_{j=1}^{J}\left[y_{i j} \log \theta_{i j}+\left(1-y_{i j}\right) \log \left(1-\theta_{i j}\right)\right]
\end{aligned}
$$

Ao considerar uma função de ligação logística, de modo geral, a probabilidade de postura do animal $i$ no dia $t_{j}$, representada por $\theta_{i j}$, é dada por:

$$
\theta_{i j}=\frac{\exp \left\{\alpha_{\mathrm{ij}}+\beta_{i j} t_{j}\right\}}{1+\exp \left\{\alpha_{\mathrm{ij}}+\beta_{i j} t_{j}\right\}},
$$

em que:

$$
\log i t\left(\theta_{i j}\right)=\alpha_{\mathrm{ij}}+\beta_{i j} t_{j}
$$
em que $\alpha_{i j}=\alpha_{i}$ : efeito aleatório do indivíduo $i$ para todas os $j$-dias; $\beta_{i j}=\beta_{i}$ : coeficientes de regressão do indivíduo $i$ para todas os $j$-dias.

Biologicamente, os parâmetros $\alpha$ e $\beta$ representam, respectivamente, o início da postura e a evolução da postura (velocidade de alcance ao pico de postura) da ave.

O logaritmo da função de verossimilhança, em função dos parâmetros $\alpha$ e $\beta$, foi dado por: 
$l(\alpha, \beta)=\sum_{i=1}^{n} \sum_{j=1}^{J} y_{i j}\left(\alpha_{i}+\beta_{i} t_{j}\right)+\sum_{i=1}^{n} \sum_{j=1}^{J}\left(1-y_{i j}\right) \log \left(1-\theta_{i j}\right)$

Para a modelagem bayesiana, foram consideradas distribuições Normais a priori para $\alpha$ e $\beta$, tal que: $\alpha_{i} \sim N\left(0, \sigma_{\alpha}^{2}\right)$ e $\beta_{i} \sim N\left(0, \sigma_{\beta}^{2}\right)$, em que os hiperparâmetros $\sigma_{\alpha}^{2}$ e $\sigma_{\beta}^{2}$ são obtidos por meio da precisão $t,(\sigma=1 / \sqrt{\tau})$, obtida por uma distribuição de probabilidade Gama a priori não informativa: $\tau \sim \operatorname{Gama}\left(10^{-3}, 10^{-3}\right)$ (valores para caracterizar uma distribuição vaga, segundo o pacote BRugs do R (R Development Core Team, 2007), software utilizado).

Médias a posteriori para os parâmetros da curva de probabilidade de postura de cada animal dada em (4) foram tomadas no primeiro estágio por meio do mesmo pacote computacional.

Para cada linhagem, nesse estágio, utilizando valores iniciais aleatórios de uma distribuição normal padronizada, foram geradas 10.000 amostras, por meio do método de Monte Carlo em Cadeias de Markov (MCMC), para os parâmetros de interesse. Foi utilizado um descarte inicial de $10 \%$ com um intervalo de amostragem de cinco iterações para eliminar a autocorrelação, com a obtenção de 1800 amostras finais.

Segundo estágio: a estrutura dos dados foi elaborada de tal forma que foi possível verificar a existência de diferenças entre os níveis de energia das dietas. Portanto, uma análise tetracaracterística foi apropriada, sendo cada parâmetro tratado como característica de interesse. Para todas as linhagens, foi utilizado um modelo animal que incluiu os efeitos do grupo de eclosão e genético aditivo.

Em uma análise tetracaracterística, foi utilizado o modelo animal descrito a seguir (Sorensen \& Gianola, 2002): $\theta_{i}=X_{i} \gamma+Z_{i} a+e_{i}$; em que $i=1,2, \ldots, \mathrm{n} ; \theta=\left[\begin{array}{lll}\theta_{11} & \theta_{12} & \theta_{21}\end{array}\right.$

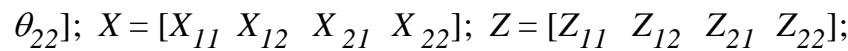
$\gamma=\left[\begin{array}{llll}\gamma_{11} & \gamma_{12} & \gamma_{21} & \gamma_{22}\end{array}\right]$ e $a=\left[\begin{array}{llll}a_{11} & a_{12} & a_{21} & a_{22}\end{array}\right]$, em que $\theta_{11}=\alpha_{1}$ e $\theta_{12}=\beta_{1}$ são os vetores de observações para os coeficientes da curva individual, dada em (4), tratados com a dieta $1(2.900 \mathrm{kcal} / \mathrm{kg} \mathrm{EM})$, e $\theta_{21}=\alpha_{2}$ e $\theta_{22}=\beta_{2}$, tratados com a dieta $2(2.500 \mathrm{kcal} / \mathrm{kg} \mathrm{EM}) ; X$ e $Z$ são matrizes de incidência dos efeitos de ambiente e genéticos, respectivamente; $\gamma, \alpha$ e $e$ são os vetores dos efeitos de ambiente, genéticos aditivos e dos erros aleatórios associados a cada observação, respectivamente.

Os resíduos foram considerados independentes entre indivíduos e normalmente distribuídos, isto é, $e_{i} \mid R_{e} \sim N\left(0, R_{\mathrm{e}}\right)$, em que $R_{\mathrm{e}}$ é a matriz de covariância residual entre as características e $a \mid A, G_{0} \sim N\left(0, G_{0} \otimes A\right)$, em que $G_{0}$ é a matriz de covariância genética entre as características e $A$, a matriz de parentesco entre os animais. Entretanto, em função do significado biológico dos parâmetros, as covariâncias residuais entre parâmetros de um mesmo indivíduo foram consideradas não nulas e entre animais com tratamentos distintos, nulas, isto é, $\operatorname{cov}\left(\mathrm{e}_{\alpha_{\mathrm{i}} \gamma_{\mathrm{j}}}\right) \neq 0$ se $i=$ $j, \mathrm{e} \operatorname{cov}\left(\mathrm{e}_{\alpha_{\mathrm{i}} \alpha_{\mathrm{j}}}\right)=\operatorname{cov}\left(\mathrm{e}_{\gamma_{\mathrm{i}} \gamma_{\mathrm{j}}}\right)=\operatorname{cov}\left(\mathrm{e}_{\alpha_{\mathrm{i}} \gamma_{\mathrm{j}}}\right)=0$ se $i^{1} j$.

$\left[\begin{array}{c}\theta \\ a \\ e\end{array}\right] \sim N M V\left\{\left[\begin{array}{c}X \gamma \\ 0 \\ 0\end{array}\right],\left[\begin{array}{ccc}V & Z G & R \\ G Z^{\prime} & G & \varnothing \\ R & \varnothing & R\end{array}\right]\right\} \quad$ em que $V=Z G Z^{\prime}+R, R=R_{e} \otimes I$ e $G=G_{0} \otimes A$;

$$
G_{0}=\left[\begin{array}{cccc}
\sigma_{\alpha_{1}}^{2} & \sigma_{\alpha_{1} \beta_{1}} & \sigma_{\alpha_{1} \alpha_{2}} & \sigma_{\alpha_{1} \beta_{2}} \\
\sigma_{\alpha_{1} \beta_{1}} & \sigma_{\beta_{1}}^{2} & \sigma_{\beta_{1} \alpha_{2}} & \sigma_{\beta_{1} \beta_{2}} \\
\sigma_{\alpha_{1} \alpha_{2}} & \sigma_{\beta_{1} \alpha_{2}} & \sigma_{\alpha_{2}}^{2} & \sigma_{\alpha_{2} \beta_{2}} \\
\sigma_{\alpha_{1} \beta_{2}} & \sigma_{\beta_{1} \beta_{2}} & \sigma_{\alpha_{2} \beta_{2}} & \sigma_{\beta_{2}}^{2}
\end{array}\right]
$$

$R_{e}=\left[\begin{array}{cccc}\sigma_{e_{\alpha_{1}}}^{2} & \sigma_{e_{\alpha_{1} \beta_{1}}} & \varnothing & \varnothing \\ \sigma_{e_{\alpha_{1} \beta_{1}}} & \sigma_{e_{\beta_{1}}}^{2} & \varnothing & \varnothing \\ \varnothing & \varnothing & \sigma_{e_{\alpha_{2}}}^{2} & \sigma_{e_{\alpha_{2} \beta_{2}}} \\ \varnothing & \varnothing & \sigma_{e_{\alpha_{2} \beta_{2}}} & \sigma_{e_{\beta_{2}}}^{2}\end{array}\right]$, em que

$\sigma_{\alpha}^{2}, \sigma_{\beta}^{2}, \sigma_{e_{\alpha,}}^{2}$ e $\sigma_{e_{\beta .}}^{2}=$ variâncias genéticas aditivas e de efeitos residuais, respectivamente, dos coeficientes da curva de probabilidade de postura de animais tratados com as dietas 1 e $2 ; \sigma_{\alpha \beta}$ e $\sigma_{e_{\alpha \beta}}=$ covariâncias genética aditiva e de efeito residual, respectivamente, entre as características analisadas.

Foi assumida, para o parâmetro de efeitos ambientais, $\gamma$, uma distribuição não- informativa constante a priori: $p(\gamma) \propto c t e$; para os componentes de covariância genética aditiva e residual, foram consideradas distribuições a priori Wishart Invertidas não-informativas:

$$
\begin{array}{r}
p\left(G_{0} \mid v_{a}, V_{a}\right) \propto\left|G_{0}\right|^{-\frac{1}{2}\left(v_{a}+k+1\right)} \exp \left\{-\frac{1}{2} \operatorname{tr}\left(G_{0}^{-1} V_{a}^{-1}\right)\right\} \\
p\left(R_{e} \mid v_{e}, V_{e}\right) \propto\left|R_{e}\right|^{-\frac{1}{2}\left(v_{e}+k+1\right)} \exp \left\{-\frac{1}{2} \operatorname{tr}\left(R_{e}^{-1} V_{e}^{-1}\right)\right\},
\end{array}
$$

sendo $k$ o número de características analisadas, hiperparâmetros $v_{a}=v_{e}<-(k+1)$ para gerar distribuições próprias e matrizes $V_{a}$ e $V_{e}$ definidas positivas.

As densidades condicionais a posteriori dos efeitos genéticos geradas são distribuições normais multivariadas $(N M V)$, definidas pelos elementos da matriz de coeficientes e do lado direito das equações de modelos mistos (Henderson, 1973): $\theta \mid . \sim N M V\left[\hat{\theta}, C^{-1}\right]$, tal que $C=M^{\prime} R^{-1} M+\Omega$ sendo $M=[X: Z]$ a matriz de incidências e $\Omega=\left[\begin{array}{cc}\phi & \phi \\ \phi & G_{o}^{-1} \otimes A^{-1}\end{array}\right]$. As densidades condicionais $a$ 
posteriori das matrizes de (co)variância seguem a forma de Wishart Invertidas $(I W): G_{0} \mid . \sim I W\left[\left(V_{a}^{-1}+S_{a}\right)^{-1}, v_{a}+q\right] \mathrm{e}$ $R_{e} \mid . \sim I W\left[\left(V_{e}^{-1}+S_{e}\right)^{-1}, v_{e}+n\right]$, em que $e$ representa o vetor de soluções para efeitos residuais: $e_{i}=\theta_{i}-X_{i} \gamma-Z_{i a}$; $S_{e}=e^{\prime} e ; S_{a}=a^{\prime} A^{-1} a ; q$ representa ordem da matriz $A ;$ e $n$, número de animais com observações.

Foram assumidos, como hiperparâmetros, valores iniciais restritos, como descritos anteriormente: $n_{a}=n_{e}=-6$;

$V_{a}=\left[\begin{array}{cccc}1 & 0,5 & 0,2 & 0,1 \\ 0,5 & 1 & 0,5 & 0,5 \\ 0,2 & 0,5 & 1 & 0,5 \\ 0,1 & 0,2 & 0,5 & 1\end{array}\right] \mathrm{e} \quad V_{e}=\left[\begin{array}{cccc}1 & 0,5 & \varnothing & \varnothing \\ 0,5 & 1 & \varnothing & \varnothing \\ \varnothing & \varnothing & 1 & 0,5 \\ \varnothing & \varnothing & 0,5 & 1\end{array}\right]$

Para cada linhagem, estimativas bayesianas dos componentes de (co)variância genética aditiva, residuais e parâmetros genéticos (médias, desvios-padrão e medianas a posteriori das distribuições marginais condicionais) foram obtidas por meio do sistema computacional MTGSAM (Multiple Trait Gibbs Sampler in Animal Model) (Van Tassel \& Van Vleck, 1996), que utilizou o método de Monte Carlo em Cadeias de Markov (MCMC) para gerar 10.000.000 amostras para os parâmetros. Foi realizado um descarte inicial de 500.000 iterações e utilizado um intervalo de amostragem de 1000 iterações para eliminar a autocorrelação, sendo obtidas 4000 amostras finais.

O monitoramento da convergência das cadeias geradas pelo amostrador de Gibbs, emambos estágios do procedimento, foi realizado por meio de análises gráficas e por testes diagnósticos de Geweke (1992) e de Heidelberger \& Welch (1983), disponíveis no CODA (Convergence Diagnosis and Output Analysis) (Spiegelhalter et al., 1994), implementado no programa R (R Development Core Team, 2007).

Foram construídos os intervalos com $95 \%$ de credibilidade (percentis da distribuição a posteriori) e as regiões de alta densidade (RAD: região de maior probabilidade da ocorrência do parâmetro), para todos os componentes de (co)variância e parâmetros genéticos estimados.

A estrutura tetracaracterística estabelecida possibilitou fazer comparações nos níveis de energia em uma linhagem específica, além de permitir comparar as (co)variâncias e a herdabilidade entre linhagens, a fim de observar diferenças significativas a $5 \%$, caso o valor zero " 0 " não estiver contido no intervalo de credibilidade do contraste $\Delta$, $\operatorname{ICr}[\Delta ; 95 \%]$.

\section{Resultados e Discussão}

As estimativas dos componentes de covariância genéticos, residuais e as herdabilidades dos parâmetros da curva de probabilidade de postura, com seus respectivos intervalos de credibilidade ( $\mathrm{ICr}$ : percentis $\mathrm{p}_{2,5 \%}-\mathrm{p}_{97,5 \%}$ ) e região de alta densidade (RAD), em nível de 95\%, foram obtidos, respectivamente, para as linhagens amarela, azul e vermelha (Tabelas 1, 2 e 3 ).

De modo geral, as distribuições dos parâmetros analisados foram simétricas, como pode ser notado pelas comparações entre ICr e RAD, assim como valores das estimativas próximas entre a média e a mediana, a posteriori.

Dentro de cada linhagem, não houve diferenças significativas entre os componentes de covariância e herdabilidades (considerando os contrastes: $\alpha_{1}-\alpha_{2}$, $\beta_{1}-\beta_{2}$ e $\left.\alpha_{1} \beta_{1}-\alpha_{2} \beta_{2}\right)$ quando as aves foram alimentadas com rações contendo diferentes níveis de energia, indicando que não houve heterogeneidade de variância.

A implementação de métodos que contemplam heterogeneidade de variância tem se baseado na utilização de modelos multicaracterísticos, em que a característica que se pretende avaliar é tratada como diferentes características em cada classe de heterogeneidade de variância. Esse método permite a estimação dos efeitos de ambiente e a predição dos valores genéticos, ponderando adequadamente as observações de acordo com as diferentes variâncias em cada classe de heterogeneidade e, ainda, considerando a estrutura de (co)variâncias entre as classes (Weigel \& Gianola, 1993; Martins, 2002b; Paula et al., 2009).

As estimativas de herdabilidades (Tabelas 1, 2 e 3), respectivamente nas linhagens amarela, azul e vermelha, para os parâmetros $\alpha$ e $\beta$ na dieta com o nível preconizado de energia, foram 0,21 e 0,$50 ; 0,12$ e 0,49 ; e 0,12 e 0,48 e, na dieta de baixa energia, 0,23 e 0,50; 0,13 e 0,50 ; e 0,10 e 0,47. Para ambos níveis de energia, as herdabilidades para o parâmetro $\beta$ foram altas, indicando que, nesse estudo, nas linhagens analisadas, há variações genéticas aditivas que permitem uma boa resposta à seleção.

Mandal et al. (1994) encontraram estimativas de herdabilidade de $18 \%$ a $26 \%$ para produção de ovos de codornas Coturnix coturnix japonica. Minvielle (1998), em revisão sobre o melhoramento genético de codornas, citam para a produção de ovos, valores entre $32 \%$ e $39 \%$ para herdabilidade, utilizando, respectivamente, métodos de estimação por meio de Mínimos Quadrados (LS) e Máxima Verossimilhança Restrita (REML), considerando um modelo animal.

Conti (2007) analisou o banco de dados do presente trabalho, utilizando procedimento frequentista por meio de Modelos Lineares Generalizados (GLM) para obtenção das estimativas dos parâmetros das curvas de cada ave e encontrou valores inferiores para a herdabilidade (energia 
Tabela 1 - Estimativas a posteriori dos componentes genéticos para os parâmetros da curva de postura na linhagem amarela

\begin{tabular}{|c|c|c|c|c|c|c|c|}
\hline Componente & Média & Desvio-padrão ${ }^{1}$ & Mediana & $2,5 \%$ & $\mathrm{p}_{97,5 \%}$ & $\mathrm{RAD}_{\mathrm{inf}}$ & $\mathrm{RAD}_{\text {sup }}$ \\
\hline & \multicolumn{7}{|c|}{ Covariância genética } \\
\hline$\alpha_{1}$ & 0,44958 & 0,29170 & 0,37799 & 0,11055 & 1,20327 & 0,06663 & 1,01261 \\
\hline$\alpha_{2}$ & 0,54790 & 0,44873 & 0,41398 & 0,09315 & 1,77161 & 0,04861 & 1,45375 \\
\hline$\alpha_{1}$ & 0,01402 & 0,00199 & 0,01386 & 0,01060 & 0,01836 & 0,01040 & 0,01802 \\
\hline$\beta_{2}$ & 0,01550 & 0,00234 & 0,01529 & 0,01149 & 0,02054 & 0,01124 & 0,02009 \\
\hline$\alpha_{1} \alpha_{2}$ & 0,30433 & 0,26659 & 0,24759 & $-0,04163$ & 0,97348 & $-0,09588$ & 0,86214 \\
\hline$\alpha_{1} \beta_{1}$ & $-0,00710$ & 0,01193 & $-0,00510$ & $-0,03565$ & 0,01099 & $-0,03202$ & 0,01294 \\
\hline$\alpha_{1} \beta_{2}$ & $-0,00660$ & 0,01241 & $-0,00519$ & $-0,03583$ & 0,01501 & $-0,03333$ & 0,01699 \\
\hline$\alpha_{2} \beta_{1}$ & 0,00126 & 0,01249 & 0,00271 & $-0,02776$ & 0,02299 & $-0,02706$ & 0,02364 \\
\hline$\alpha_{2} \beta_{2}$ & $-0,01240$ & 0,01609 & $-0,00869$ & $-0,05352$ & 0,00924 & $-0,04810$ & 0,01224 \\
\hline$b_{1} \beta_{2}$ & 0,00428 & 0,00139 & 0,00423 & 0,00175 & 0,00715 & 0,00167 & 0,00704 \\
\hline$\alpha_{1}-\alpha_{2}$ & $-0,09830$ & 0,47367 & $-0,02862$ & $-1,28731$ & 0,69770 & $-1,11893$ & 0,82684 \\
\hline$\beta_{1}-\beta_{2}^{2}$ & $-0,00150$ & 0,00299 & $-0,00144$ & $-0,00740$ & 0,00426 & $-0,00740$ & 0,00423 \\
\hline$\alpha_{1} \beta_{1}-\alpha_{2} \beta_{2}$ & 0,00520 & 0,01905 & 0,00349 & $-0,02886$ & 0,04890 & $-0,03111$ & 0,04589 \\
\hline \multicolumn{8}{|c|}{ Covariância residual } \\
\hline$\alpha_{1}$ & 1,63600 & 0,31958 & 1,64667 & 0,97068 & 2,24346 & 1,01179 & 2,28116 \\
\hline$\alpha_{1} \beta_{1}$ & $-0,03870$ & 0,01757 & $-0,03828$ & $-0,07530$ & $-0,00548$ & $-0,07254$ & $-0,00292$ \\
\hline$\beta_{1}$ & 0,01423 & 0,00197 & 0,01406 & 0,01087 & 0,01852 & 0,01073 & 0,01830 \\
\hline$\alpha_{2}$ & 1,79246 & 0,45708 & 1,83759 & 0,73815 & 2,57621 & 0,84364 & 2,63856 \\
\hline$\alpha_{2} \beta_{2}$ & $-0,03540$ & 0,02176 & $-0,03445$ & $-0,08045$ & 0,00402 & $-0,07944$ & 0,00457 \\
\hline$\beta_{2}$ & 0,01542 & 0,00228 & 0,01523 & 0,01151 & 0,02040 & 0,01134 & 0,02014 \\
\hline$\alpha_{1}-\alpha_{2}$ & $-0,15650$ & 0,53344 & $-0,18655$ & $-1,12084$ & 0,99942 & $-1,14940$ & 0,96349 \\
\hline$\beta_{1}-\beta_{2}$ & $-0,00120$ & 0,00302 & $-0,00116$ & $-0,00731$ & 0,00465 & $-0,00696$ & 0,00495 \\
\hline$\alpha_{1} \beta_{1}-\alpha_{2} \beta_{2}$ & $-0,00330$ & 0,02744 & $-0,00376$ & $-0,05517$ & 0,05236 & $-0,05387$ & 0,05333 \\
\hline \multicolumn{8}{|c|}{ Herdabilidade } \\
\hline$\alpha_{1}$ & 0,21405 & 0,12885 & 0,18401 & 0,05302 & 0,53024 & 0,03505 & 0,46803 \\
\hline$\alpha_{2}$ & 0,23140 & 0,17363 & 0,18115 & 0,03940 & 0,69542 & 0,02366 & 0,59454 \\
\hline$\beta_{1}$ & 0,49614 & 0,04369 & 0,49547 & 0,41199 & 0,58180 & 0,41146 & 0,58092 \\
\hline$\beta_{2}$ & 0,50127 & 0,04627 & 0,50082 & 0,41306 & 0,59164 & 0,41422 & 0,59264 \\
\hline$\alpha_{1-} \alpha_{2}$ & $-0,01743$ & 0,19321 & 0,00375 & $-0,47329$ & 0,32714 & $-0,45660$ & 0,34277 \\
\hline$\beta_{1-} \beta_{2}$ & $-0,00515$ & 0,06318 & $-0,00554$ & $-0,12849$ & 0,11835 & $-0,12548$ & 0,12100 \\
\hline
\end{tabular}

$\mathrm{p}_{2,5 \%}$ e $\mathrm{p}_{97,5 \%}=$ intervalo de $95 \%$ de credibilidade; $\mathrm{RAD}_{\text {inf }}$ e $\mathrm{RAD}_{\text {sup }}=$ intervalo de alta densidade.

preconizada para $\alpha: 0,003 ; 0,002$ e 0,282 ; para $\beta: 0,065$; 0,034 e 0,379 ; baixa energia para $\alpha: 0,003 ; 0,004$ e 0,442 , e para $\beta: 0,048 ; 0,514$ e 0,502 para as linhagens amarela, azul e vermelha, respectivamente).

As correlações genéticas dos parâmetros $\alpha$ e $\beta$ entre níveis de energia, para as três linhagens (Tabela 4) indicam a presença de interação genótipo $\times$ ambiente, que é mais intensa para o parâmetro $\beta$ em comparação a $\alpha$. Assim, a seleção para esses parâmetros no ambiente de alta energia resultará em menor ganho genético; isso se os animais forem criados no ambiente de menor energia em vez de no ambiente de alta energia.

Conti (2007) relata que a seleção seria mais eficiente se realizada considerando a dieta de baixa energia em todas as linhagens, e conclui, por análise de correlações genéticas entre as dietas, que existe forte interação genótipo $\times$ ambiente para o parâmetro $\beta$ na linhagem azul, recomendando que a progênie seja criada no mesmo ambiente (dieta) nos quais os pais foram selecionados para que se obtenha o ganho genético esperado. Afirma que, na linhagem vermelha, a seleção pode ser praticada considerando uma dieta de maior energia e a progênie criada em baixa energia sem que haja prejuízos na expressão das características da curva de probabilidade de postura. Conclui que a seleção seria mais eficiente se praticada na direção da característica velocidade de alcance ao pico de postura, $\beta$, considerando uma dieta de baixa energia.

Alguns experimentos têm demonstrado que o comportamento das aves pode ser modificado por meio da seleção e que interações genótipo $\times$ ambiente são comuns para muitos comportamentos (Ton, 2004).

Houve diferença entre todas linhagens de codornas de postura quanto à herdabilidade dos parâmetros das curvas, o que indica diferentes potenciais de respostas à seleção para a produção de ovos (Tabela 5). Entretanto, a linhagem amarela apresenta maior herdabilidade para o parâmetros $\alpha$, o que indica maior potencial para mudanças por seleção para a produção na fase inicial de postura. Baseado no parâmetro $\beta$ (velocidade de alcance do pico de postura), as três linhagens apresentam o mesmo potencial.

Considerando as estimativas dos componentes de covariância genética aditiva entre as constantes das curvas 
Tabela 2 - Estimativas a posteriori dos componentes genéticos para os parâmetros da curva de postura na linhagem azul

\begin{tabular}{|c|c|c|c|c|c|c|c|}
\hline Componente & Média & Desvio-padrão & Mediana & $\mathrm{p}_{2,5 \%}$ & $\mathrm{p}_{97,5 \%}$ & $\mathrm{RAD}_{\text {inf }}$ & $\mathrm{RAD}_{\text {sup }}$ \\
\hline \multicolumn{8}{|c|}{ Covariância genética } \\
\hline$\alpha_{1}$ & 0,36879 & 0,23731 & 0,30652 & 0,09689 & 0,99525 & 0,06407 & 0,84267 \\
\hline$\alpha_{2}$ & 0,33323 & 0,22431 & 0,27144 & 0,08016 & 0,91365 & 0,04884 & 0,77713 \\
\hline$\beta_{1}^{2}$ & 0,01274 & 0,00170 & 0,01261 & 0,00983 & 0,01647 & 0,00971 & 0,01627 \\
\hline$\beta_{2}$ & 0,01184 & 0,00154 & 0,01170 & 0,00922 & 0,01522 & 0,00897 & 0,01489 \\
\hline$\alpha_{1} \alpha_{2}$ & 0,22794 & 0,18366 & 0,18416 & $-0,00606$ & 0,70404 & $-0,03739$ & 0,62156 \\
\hline$\alpha_{1} \beta_{1}$ & $-0,00601$ & 0,00979 & $-0,00449$ & $-0,02964$ & 0,00908 & $-0,02624$ & 0,01153 \\
\hline$\alpha_{1} \beta_{2}$ & $-0,00401$ & 0,00803 & $-0,00329$ & $-0,02191$ & 0,01003 & $-0,02129$ & 0,01048 \\
\hline$\alpha_{2} \beta_{1}$ & 0,00160 & 0,00861 & 0,00243 & $-0,01856$ & 0,01670 & $-0,01587$ & 0,01859 \\
\hline$\alpha_{2} \beta_{2}$ & $-0,00530$ & 0,00815 & $-0,00404$ & $-0,02511$ & 0,00764 & $-0,02269$ & 0,00919 \\
\hline$\beta_{1} \beta_{2}$ & 0,00352 & 0,00106 & 0,00348 & 0,00150 & 0,00567 & 0,00147 & 0,00562 \\
\hline$\alpha_{1}-\alpha_{2}$ & 0,03555 & 0,25967 & 0,03084 & $-0,49031$ & 0,58610 & $-0,50316$ & 0,57110 \\
\hline$\beta_{1}-\beta_{2}$ & 0,00090 & 0,00224 & 0,00087 & $-0,00351$ & 0,00538 & $-0,00345$ & 0,00542 \\
\hline$\alpha_{1} \beta_{1}-\alpha_{2} \beta_{2}$ & $-0,00071$ & 0,01196 & $-0,00057$ & $-0,02566$ & 0,02273 & $-0,02559$ & 0,02278 \\
\hline \multicolumn{8}{|c|}{ Covariância residual } \\
\hline$\alpha_{1}$ & 2,57757 & 0,34995 & 2,57535 & 1,89276 & 3,28045 & 1,90182 & 3,28684 \\
\hline$\alpha_{1} \beta_{1}$ & $-0,05667$ & 0,01951 & $-0,05592$ & $-0,09656$ & $-0,02022$ & $-0,09564$ & $-0,01967$ \\
\hline$\beta_{1}$ & 0,01343 & 0,00177 & 0,01331 & 0,01035 & 0,01734 & 0,01013 & 0,01702 \\
\hline$\alpha_{2}$ & 2,15858 & 0,31735 & 2,15900 & 1,52574 & 2,77332 & 1,55217 & 2,79738 \\
\hline$\alpha_{2} \beta_{2}$ & $-0,03686$ & 0,01665 & $-0,03603$ & $-0,07161$ & $-0,00618$ & $-0,07012$ & $-0,00504$ \\
\hline$\beta_{2}$ & 0,01195 & 0,00154 & 0,01183 & 0,00924 & 0,01529 & 0,00907 & 0,01501 \\
\hline$\alpha_{1}-\alpha_{2}$ & 0,41900 & 0,44041 & 0,41653 & $-0,44801$ & 1,29740 & $-0,47290$ & 1,26149 \\
\hline$\beta_{1}-\beta_{2}^{2}$ & 0,00148 & 0,00234 & 0,00145 & $-0,00307$ & 0,00617 & $-0,00319$ & 0,00598 \\
\hline$\alpha_{1} \beta_{1}-\alpha_{2} \beta_{2}$ & $-0,01981$ & 0,02512 & $-0,01981$ & $-0,06950$ & 0,02838 & $-0,07102$ & 0,02652 \\
\hline \multicolumn{8}{|c|}{ Herdabilidade } \\
\hline$\alpha_{1}$ & 0,12492 & 0,07739 & 0,10539 & 0,03294 & 0,32575 & 0,02409 & 0,28302 \\
\hline & 0,13392 & 0,08730 & 0,10957 & 0,03136 & 0,35780 & 0,01895 & 0,30603 \\
\hline$\beta_{J}$ & 0,48694 & 0,04145 & 0,48688 & 0,40641 & 0,56931 & 0,40323 & 0,56590 \\
\hline$\beta_{2}$ & 0,49768 & 0,03974 & 0,49772 & 0,42034 & 0,57577 & 0,42029 & 0,57565 \\
\hline$\alpha_{1-} \alpha_{2}$ & $-0,00901$ & 0,09297 & $-0,00483$ & $-0,21299$ & 0,17550 & $-0,21680$ & 0,17000 \\
\hline$\beta_{1} \beta_{2}$ & $-0,01072$ & 0,05692 & $-0,01096$ & $-0,12111$ & 0,10103 & $-0,12253$ & 0,09956 \\
\hline
\end{tabular}

$\mathrm{p}_{2,5 \%}$ e $\mathrm{p}_{97,5 \%}=$ intervalo de $95 \%$ de credibilidade; $\mathrm{RAD}_{\text {inf }}$ e $\mathrm{RAD}_{\text {sup }}=$ intervalo de alta densidade.

Tabela 3 - Estimativas a posteriori dos componentes genéticos para os parâmetros da curva de postura na linhagem vermelha

\begin{tabular}{|c|c|c|c|c|c|c|c|}
\hline & Média & Desvio-padrão & Mediana & $\mathrm{p}_{2,5 \%}$ & $\mathrm{p}_{97,5 \%}$ & RAD $_{\text {inf }}$ & $\mathrm{RAD}_{\text {sup }}$ \\
\hline \multicolumn{8}{|c|}{ Covariância genética } \\
\hline$\alpha_{1}$ & 0,40950 & 0,29036 & 0,32724 & 0,09526 & 1,21924 & 0,06512 & 1,01244 \\
\hline$\alpha_{2}$ & 0,45856 & 0,44521 & 0,31118 & 0,08059 & 1,67324 & 0,04069 & 1,31407 \\
\hline$\beta_{1}^{2}$ & 0,01206 & 0,00156 & 0,01195 & 0,00936 & 0,01547 & 0,00919 & 0,01524 \\
\hline$\beta_{2}$ & 0,01336 & 0,00185 & 0,01320 & 0,01021 & 0,01744 & 0,00994 & 0,01705 \\
\hline$\alpha_{1} \alpha_{2}$ & 0,22902 & 0,24150 & 0,17042 & $-0,09856$ & 0,85258 & $-0,15488$ & 0,76965 \\
\hline$\alpha_{1} \beta_{1}$ & $-0,00843$ & 0,01097 & $-0,00621$ & $-0,03604$ & 0,00703 & $-0,03199$ & 0,00884 \\
\hline$\alpha_{1} \beta_{2}$ & $-0,00494$ & 0,01069 & $-0,00363$ & $-0,03027$ & 0,01350 & $-0,02760$ & 0,01563 \\
\hline$\alpha_{2} \beta_{1}^{2}$ & $-0,00032$ & 0,01053 & 0,00125 & $-0,02569$ & 0,01719 & $-0,02202$ & 0,01945 \\
\hline$\alpha_{2} \beta_{2}$ & $-0,01015$ & 0,01456 & $-0,00660$ & $-0,04785$ & 0,00733 & $-0,04018$ & 0,01030 \\
\hline$\beta_{1} \beta_{2}$ & 0,00376 & 0,00111 & 0,00373 & 0,00168 & 0,00601 & 0,00164 & 0,00597 \\
\hline$\alpha_{1}-\alpha_{2}$ & $-0,04907$ & 0,49380 & 0,00906 & $-1,27064$ & 0,79565 & $-1,13005$ & 0,91932 \\
\hline$\beta_{1}^{1}-\beta_{2}^{2}$ & $-0,00130$ & 0,00236 & $-0,00124$ & $-0,00603$ & 0,00313 & $-0,00579$ & 0,00331 \\
\hline$\alpha_{1} \beta_{1}-\alpha_{2} \beta_{2}$ & 0,00173 & 0,01739 & 0,00044 & $-0,02968$ & 0,04133 & $-0,03211$ & 0,03745 \\
\hline \multicolumn{8}{|c|}{ Covariância residual } \\
\hline$\alpha_{1}$ & 3,19679 & 0,42899 & 3,19802 & 2,35421 & 4,05451 & 2,34310 & 4,03081 \\
\hline$\alpha_{1} \beta_{1}$ & $-0,07933$ & 0,02163 & $-0,07833$ & $-0,12402$ & $-0,03904$ & $-0,12111$ & $-0,03651$ \\
\hline$\beta_{1}{ }^{1}$ & 0,01332 & 0,00169 & 0,01322 & 0,01035 & 0,01702 & 0,01007 & 0,01661 \\
\hline$\alpha_{2}$ & 3,95600 & 0,61853 & 3,97791 & 2,64798 & 5,11882 & 2,71360 & 5,16136 \\
\hline$\alpha_{2} \beta_{2}$ & $-0,09608$ & 0,02820 & $-0,09524$ & $-0,15441$ & $-0,04301$ & $-0,15087$ & $-0,04079$ \\
\hline$\beta_{2}$ & 0,01509 & 0,00208 & 0,01493 & 0,01148 & 0,01967 & 0,01133 & 0,01938 \\
\hline$\alpha_{1}-\alpha_{2}$ & $-0,75921$ & 0,73842 & $-0,77507$ & $-2,15239$ & 0,74647 & $-2,17351$ & 0,71443 \\
\hline$\beta_{1}-\beta_{2}^{2}$ & $-0,00177$ & 0,00267 & $-0,00174$ & $-0,00711$ & 0,00341 & $-0,00730$ & 0,00319 \\
\hline$\alpha_{1} \beta_{1}-\alpha_{2} \beta_{2}$ & 0,01675 & 0,03523 & 0,01647 & $-0,05060$ & 0,08714 & $-0,05060$ & 0,08716 \\
\hline \multicolumn{8}{|c|}{ Herdabilidade } \\
\hline & 0,11349 & 0,07792 & 0,09184 & 0,02622 & 0,32578 & 0,01593 & 0,27291 \\
\hline$\alpha_{2}^{1}$ & 0,10411 & 0,09709 & 0,07159 & 0,01790 & 0,37208 & 0,01047 & 0,30049 \\
\hline$\beta_{l}^{2}$ & 0,47536 & 0,04121 & 0,47472 & 0,39570 & 0,55753 & 0,39310 & 0,55419 \\
\hline$\beta_{2}$ & 0,46975 & 0,04410 & 0,46890 & 0,38589 & 0,55739 & 0,38550 & 0,55659 \\
\hline$\alpha_{1-} \alpha_{2}$ & 0,00940 & 0,11523 & 0,01602 & $-0,27157$ & 0,22985 & $-0,23883$ & 0,26023 \\
\hline$\beta_{1-} \beta_{2}$ & 0,00560 & 0,05932 & 0,00506 & $-0,11188$ & 0,12127 & $-0,11315$ & 0,11971 \\
\hline
\end{tabular}

$\mathrm{p}_{2,5 \%}$ e $\mathrm{p}_{97,5 \%}=$ intervalo de $95 \%$ de credibilidade; $\mathrm{RAD}_{\text {inf }}$ e $\mathrm{RAD}_{\text {sup }}=$ intervalo de alta densidade. 
Tabela 4 - Médias a posteriori da correlação genética para os coeficientes das curvas de probabilidade de postura em dois níveis de energia

\begin{tabular}{lccccc}
\hline Linhagem & & \multicolumn{4}{c}{ Característica } \\
\cline { 3 - 6 } & & $\alpha_{1}$ & $\alpha_{2}$ & $\beta_{1}$ & $\beta_{2}$ \\
\hline Amarela & $\alpha_{1}$ & - & 0,613 & $-0,089$ & $-0,079$ \\
& $\alpha_{2}$ & & - & 0,014 & $-0,135$ \\
& $\beta_{1}$ & & & - & 0,290 \\
& $\beta_{2}$ & & & & - \\
\multirow{4}{*}{ Azul } & $\alpha_{1}$ & - & 0,650 & $-0,088$ & $-0,061$ \\
& $\alpha_{2}$ & & - & 0,246 & $-0,084$ \\
& $\beta_{1}$ & & & - & 0,287 \\
& $\beta_{2}$ & & & & - \\
Vermelha & $\alpha_{1}$ & - & 0,529 & $-0,120$ & $-0,067$ \\
& $\alpha_{2}$ & & - & $-0,005$ & $-0,130$ \\
& $\beta_{1}$ & & & - & 0,296 \\
& $\beta_{2}$ & & & & - \\
\hline
\end{tabular}

nos diferentes níveis de energia $\left(a_{1} a_{2}\right)$, as linhagens amarela e vermelha diferiram, mostrando que essas duas linhagens apresentam diferentes alterações nos efeitos genéticos que afetam o início da postura quando há mudança no nível de energia da ração.

As três linhagens diferiram entre si quanto à estimativa da covariância genética aditiva, entre os parâmetros lineares da curva de probabilidade de postura em diferentes níveis de energia $\left(\beta_{1} \beta_{2}\right)$, apresentando diferentes alterações nos efeitos genéticos que determinam a evolução da postura quando há mudança no nível de energia da ração.

A estimativa de covariância genética aditiva entre os parâmetros da curva de probabilidade de postura em energia preconizada $\left(\alpha_{1} \beta_{1}\right)$, na linhagem vermelha, foi diferente da amarela e da azul, entretanto, em baixa energia $\left(\alpha_{2} \beta_{2}\right)$, tal covariância apenas diferiu entre azul e vermelha. Nessas duas situações, a linhagem vermelha apresentou menor associação entre os efeitos genéticos que determinam o início e a evolução da postura.

Com relação à variação residual, apenas as linhagens amarela e azul não apresentam diferenças significativas, em ambiente com baixo nível de energia $\left(\alpha_{2} \beta_{2}\right)$.

Tabela 5 - Comparações a posteriori entre linhagens, considerando covariâncias genéticas, residuais e herdabilidades

\begin{tabular}{|c|c|c|c|c|c|c|}
\hline Parâmetro & Linhagem & Média & Desvio-padrão & Mediana & $\mathrm{p}_{2,5 \%}$ & $\mathrm{p}_{97,5 \%}$ \\
\hline \multirow{3}{*}{$\alpha_{1} \alpha_{2}$} & & & Covariância genética & & & \\
\hline & Amarela $\times$ azul & 0,07638 & 0,08466 & 0,06344 & $-0,03615$ & 0,26970 \\
\hline & Amarela $\times$ vermelha & $0,07531^{*}$ & 0,03190 & 0,07811 & 0,03583 & 0,11916 \\
\hline \multirow{3}{*}{$\alpha_{1} \beta_{1}$} & Azul $\times$ vermelha & $-0,00108$ & 0,06045 & 0,01384 & $-0,14864$ & 0,09288 \\
\hline & Amarela $\times$ azul & $-0,00109$ & 0,00217 & $-0,00061$ & $-0,00603$ & 0,00192 \\
\hline & Amarela $\times$ vermelha & $0,00132^{*}$ & 0,00119 & 0,00110 & 0,00018 & 0,00399 \\
\hline \multirow{2}{*}{$\alpha_{1} \beta_{2}$} & Azul $\times$ vermelha & $0,00241^{*}$ & 0,00136 & 0,00184 & 0,00146 & 0,00639 \\
\hline & Amarela $\times$ vermelha & $-0,00166$ & 0,00175 & $-0,00156$ & $-0,00556$ & 0,00148 \\
\hline \multirow{3}{*}{$\alpha_{2} \beta_{1}$} & Azul $\times$ vermelha & 0,00092 & 0,00268 & 0,00036 & $-0,00357$ & 0,00838 \\
\hline & Amarela $\times$ azul & $-0,00034$ & 0,00391 & 0,00028 & $-0,00920$ & 0,00627 \\
\hline & Amarela $\times$ vermelha & 0,00158 & 0,00202 & 0,00146 & $-0,00215$ & 0,00577 \\
\hline \multirow{3}{*}{$\alpha_{2} \beta_{2}$} & Azul $\times$ vermelha & 0,00191 & 0,00203 & 0,00118 & $-0,00036$ & 0,00710 \\
\hline & Amarela $\times$ azul & $-0,00705$ & 0,00803 & $-0,00465$ & $-0,02839$ & 0,00161 \\
\hline & Amarela $\times$ vermelha & $-0,00220$ & 0,00242 & $-0,00204$ & $-0,00647$ & 0,00207 \\
\hline \multirow{3}{*}{$b_{1} \beta_{2}$} & Azul $\times$ vermelha & $0,00485^{*}$ & 0,00678 & 0,00256 & 0,00011 & 0,02280 \\
\hline & Amarela $\times$ azul & $0,00076^{*}$ & 0,00033 & 0,00074 & 0,00024 & 0,00148 \\
\hline & Azul $\times$ vermelha & $-0,00024^{*}$ & 0,00006 & $-0,00024$ & $-0,00033$ & $-0,00016$ \\
\hline \multirow{4}{*}{$\alpha_{1} \beta_{1}$} & & & Covariância residual & & & \\
\hline & Amarela $\times$ azul & $0,01797^{*}$ & 0,00197 & 0,01762 & 0,01467 & 0,02144 \\
\hline & Amarela $\times$ vermelha & $0,04063^{*}$ & 0,00407 & 0,04005 & 0,03358 & 0,04873 \\
\hline & Azul $\times$ vermelha & $0,02266^{*}$ & 0,00215 & 0,02241 & 0,01909 & 0,02748 \\
\hline \multirow[t]{3}{*}{$\alpha_{2} \beta_{2}$} & Amarela $\times$ azul & 0,00142 & 0,00512 & 0,00159 & $-0,00882$ & 0,01032 \\
\hline & Amarela $\times$ vermelha & $0,06063^{*}$ & 0,00648 & 0,06078 & 0,04722 & 0,07409 \\
\hline & Azul $\times$ vermelha & $0,05922^{*}$ & 0,01156 & 0,05919 & 0,03701 & 0,08281 \\
\hline \multirow{4}{*}{$\alpha_{1}$} & & & Herdabilidade & & & \\
\hline & Amarela $\times$ azul & $0,08118^{*}$ & 0,01849 & 0,08062 & 0,05332 & 0,11638 \\
\hline & Amarela $\times$ vermelha & $0,09290^{*}$ & 0,01831 & 0,09406 & 0,06285 & 0,11880 \\
\hline & Azul $\times$ vermelha & $0,01172^{*}$ & 0,00311 & 0,01192 & 0,00233 & 0,01534 \\
\hline \multirow[t]{3}{*}{$\alpha_{2}$} & Amarela $\times$ azul & $0,08199^{*}$ & 0,03324 & 0,07358 & 0,04562 & 0,16271 \\
\hline & Amarela $\times$ vermelha & $0,11358^{*}$ & 0,02935 & 0,11215 & 0,07286 & 0,16136 \\
\hline & Azul $\times$ vermelha & $0,03159^{*}$ & 0,01165 & 0,03512 & 0,00037 & 0,03979 \\
\hline$\beta_{1}$ & Azul $\times$ vermelha & $0,01165^{*}$ & 0,00119 & 0,01165 & 0,00969 & 0,01373 \\
\hline \multirow{3}{*}{$\beta_{2}$} & Amarela $\times$ azul & $0,00362^{*}$ & 0,00141 & 0,00359 & 0,00106 & 0,00510 \\
\hline & Amarela $\times$ vermelha & $0,03177^{*}$ & 0,00145 & 0,03152 & 0,02948 & 0,03411 \\
\hline & Azul $\times$ vermelha & $0,02815^{*}$ & 0,00074 & 0,02803 & 0,02738 & 0,02925 \\
\hline
\end{tabular}

*Diferença significativa a $5 \%$ por meio de comparações bayesianas; $\mathrm{p}_{2,5 \%}$ e $\mathrm{p}_{97,5 \%}=$ intervalo de $95 \%$ de credibilidade. 
Georg et al. (2004) analisaram as mesmas linhagens desse estudo e concluíram, para a característica "peso do ovo", que as três linhagens de codornas foram diferentes, sendo a linhagem amarela a de melhor resposta. A linhagem vermelha apresenta baixo potencial de resposta à seleção para a mesma característica e, para a característica "espessura da casca de ovo", as três linhagens apresentaram potencial semelhante de resposta à seleção

Móri et al. (2005) analisaram codornas de postura por meio de modelos lineares e concluíram que, para produção de ovos, não foram detectadas diferenças estatísticas entre quatro grupos genéticos, embora dois deles tenham apresentado discreta tendência de superioridade na porcentagem média de produção em relação aos demais grupos. Complementam que esse resultado é similar ao relatado por Altan et al. (1998), já que não encontraram diferenças significativas para produção de ovos de linhagens selecionadas para "alto ganho de peso". Outros estudos, como os de Minvielle (2004) e Piccinin et al. (2004), mostram que não encontraram diferenças para "produção de ovos" entre as mesmas linhagens estudadas.

Convém ressaltar que diferenças genéticas entre aves utilizadas em diferentes investigações, em análises de diferentes características e metodologias, podem ocasionar diferentes conclusões.

Os resultados aqui encontrados são pertinentes ao período de postura inicial (90 dias), visto que a seleção nessas linhagens é praticada com base na produção parcial, com vistas a reduzir o intervalo de geração, promovendo, assim, maior ganho genético por unidade de tempo. Dessa forma, para inferências além desse período, o modelo ajustado deveria incluir um termo quadrático, visto que a postura entra em declínio no final do período produtivo. O resultado da inclusão de mais um parâmetro provavelmente alteraria as estimativas dos componentes de (co)variâncias.

\section{Conclusões}

As linhagens de codornas de postura avaliadas diferem entre si quanto à herdabilidade dos parâmetros das curvas de probabilidade de postura, o que indica diferentes potenciais de resposta à seleção para a produção de ovos. Entretanto, para as mesmas estimativas, não há diferenças significativas entre os níveis de energia dentro de cada linhagem analisada, logo não há heterogeneidade de variância. Além disso, os valores de correlações genéticas indicam haver interação genótipo $\times$ ambiente, portanto, a seleção resultará em menor ganho genético se os animais forem criados com baixa energia dietética. Mudanças na produção de ovos podem ser obtidas por seleção com base no parâmetro $\beta$ nas três linhagens e com base no parâmetro $\alpha$ apenas na linhagem amarela.

\section{Agradecimentos}

Ao programa de Pós-graduação do Departamento de Zootecnia e ao Departamento de Estatística da Universidade Estadual de Maringá.

\section{Referências}

ALTAN, O.; OGUZ, I.; AKBAS, Y. Effects of selection for body weight and age of hen on egg characteristics in japanese quail (Coturnix coturnix japonica). Turkish Journal Veterinary Animal Sciences, v.22, n.6, p.467-473, 1998

CONTI, A.C.M. Análise genética da probabilidade diária de postura em três linhagens de codornas. 44f. 2007. Dissertação (Mestrado em Zootecnia) - Universidade Estadual de Maringá, Maringá.

GEORG, P.C. PAIVA, E.; RESENDE, R.O. et al. Estimativa de parâmetros genéticos e fenotípicos para características de produção e qualidade de ovo, em codornas de postura. In: REUNIÃO ANUAL DA SOCIEDADE BRASILEIRA DE ZOOTECNIA, 41., 2004, Campo Grande. Anais... Campo Grande: SBZ, 2004. p.1-4.

GEWEKE, J. Evaluating the accuracy of sampling-based approaches to the calculation of posterior moments (with discussion). In: Bayesian Statistics, 4. Oxford: Oxford University Press, 1992. p. 169-193.

GIANOLA, D.; FERNANDO, R.L. Bayesian methods in animal breeding theory. Journal of Animal Science, v.63, p.217-277, 1986.

HEIDELBERGER, P.; WELCH, P. Simulation run length control in the presence of an initial transient. Operations Research, v.31, p.1109-1144, 1983

HENDERSON, C.R. Sire evaluation and genetic trends. In: ANIMAL BREEDING AND GENETICS SYMPOSIUM IN HONOR OF J. LUSH, 1973, Champaign. Proceedings... Champaingn: American Society of Animal Science, 1973. p.10-41.

KRANIS, A.; SU, G.; SORENSEN, D.A. et al. The application of random regression Models in the genetic analysis of monthly egg production in Turkeys and a comparison with alternative longitudinal models. Poultry Science, v.86, p.470-475, 2007.

MANDAL, K.G., SINEA, R.; MISHRA, S.K. et al. Estimates of genetic parameters for some egg production trails in japanese quail (Coturnix coturnix japonica). Indian Journal of Animal Health, v.33, n.1, p.49-54, 1994.

MARTINS, E.N. Prospects of quail genetic breeding in Brazil. In: INTERNATIONAL SYMPOSIUM ON QUAIL PRODUCTION, 1., 2002, Lavras. Proceedings... Lavras: NECTA-DZOUniversidade Federal de Lavras, 2002a. p.205-208.

MARTINS, E.N. Avaliação genética e heterogeneidade de variâncias. In: REUNIÃO ANUAL DA SOCIEDADE BRASILEIRA DE ZOOTECNIA, 39., 2002, Recife. Anais... Recife: Sociedade Brasileira de Zootecnia, 2002b. p.205-214.

MINVIELLE, F. Genetics and breeding of Japanese quail for production around the world. In: ASIAN PACIFIC POULTRY CONGRESS, 6., 1998, Nagoia. Proceedings... Nagoia: Poultry Science Association, 1998. p.122-127.

MINVIELLE, F. The future of Japanese quail for research and production. World's Poultry Science Journal, v.60, p.50-507, 2004

MÓRI, C.; GARCIA, E.A.; PAVAN, A.C. et al. Desempenho e qualidade dos ovos de codornas de quatro grupos genéticos. Revista Brasileira de Zootecnia, v.34, n.3, p.864-869, 2005 
NELDER, J.A.; WEDDERBURN, R.W.M. Generalized linear models. Journal of the Royal Statistical Society A, v.135, n. 3 , p.370-384, 1972 .

NATIONAL RESEARCH COUNCIL - NRC. Nutrient requirements of poultry. 9.ed.rev. Washington, D.C.: National Academy Press, 1994. 155p.

PATTERSON, H.D.; THOMPSON, R. Recovery of inter-block information when block sizes are unequal. Biometrika, v.58, p.545-554, 1971

PAULA, M.C.; MARTINS, E.N.; SILVA, L.O.C. et al. Interação genótipo $\times$ ambiente para produção de leite de bovinos da raça Holandesa entre bacias leiteiras no estado do Paraná. Revista Brasileira de Zootecnia, v.38, n.3, p.467-473, 2009.

PICCININ, A.; MALHADO, C.H.M.; MORI, C. et al. Análise da curva de postura de três linhagens de codorna utilizando a técnica não paramétrica Kernel. In: SIMPÓSIO DA SOCIEDADE BRASILEIRA DE MELHORAMENTO ANIMAL, 5., 2004, Pirassununga. Proceedings... Pirassununga: Sociedade Brasileira de Zootecnia, 2004. p.1-4.

$\mathrm{R}$ Development Core Team. R: A language and environment for statistical computing. $\mathrm{R}$ Foundation for Statistical Computing, Vienna, Austria. ISBN 3-900051-07-0. Versão 2.07. Disponível em <http://www.R-project.org〉, 2007. 60p. Acesso em: 7/2/2007.

RESENDE, M.D.V. Genética biométrica e estatística no melhoramento de plantas perenes. Brasília: Embrapa Informação Tecnológica, 2002. 975p.

SAPP, R.L.; REKAYA, R.; MISZTAL, I. et al. Longitudinal multipletrait versus cumulative single-trait analysis of male and female fertility and hatchability in chickens. research note. Poultry Science, v.84, p.1010-1014, 2005.

SCHAEFFER, L.R. Application of random regression models animal breeding. Livestock Production Science, v.86, p.35-45, 2004

SORENSEN, D.; GIANOLA, D. Likelihood, Bayesian and MCMC methods in quantitative genetics. New York: Springer, 2002, 740p.

SPIEGELHALTER, D.J.; THOMAS, A.; BEST, N. et al. BUGS Bayesian inference using Gibbs sampling. Cambridge: MRC Biostatistics Unit, 1994. 60p. Versão 1.14. Disponível em <www.mrc-bsu.cam.ac.uk/bugs/winbugs/manual14.pdf〉. Acesso em: 7/2/2007.

TON, A.P.S.; MARTINS, E.N.; SAKAGUTI, E.S. et al. Estimativa da interação genótipo $x$ ambiente na produção de ovos de codornas de postura. In: SIMPÓSIO INTERNACIONAL, 2 ; CONGRESSO BRASILEIRO DE COTURNICULTURA, 1. 2004, Lavras. Anais... Lavras: UFLA, 2004. p.235.

VAN TASSEL, C.P.; VAN VLECK, L.D. Multiple trait Gibbs Sampler for animal models: flexible programs for Bayesian and likelihood-based (co)variance components inference. Journal of Animal Science, v.74, p.2586-2597, 1996.

WANG, C.S.; GIANOLA, D.; SORENSEN, D.A. et al. Response to selection for litter size in Danish Landrace pigs: a Bayesian analysis. Theory Applied Genetics, v.88, n.2, p.220-230, 1994.

WEIGEL, K.A.; GIANOLA, D. A computationally simple Bayesian method for estimation of heterogeneous withinherd phenotypic variances. Journal of Dairy Science, v.76, n.5, p.1455-1465, 1993. 